for reducing thrombotic risk and fatigue in jSLE patients. Further studies are needed to address this issue.

\section{P4 ANTIPHOSPHOLIPID SYNDROME IN PATIENTS WITH ACUTE MYOCARDIAL INFARCTION}

${ }^{1}$ Mikel Jordhani, ${ }^{2}$ Dorina Ruci, ${ }^{3}$ Joana Seiti. ${ }^{1}$ Dept. Internal Medicine, Rheumatology Clinic, UHC Mother Teresa, Tirana; ${ }^{2}$ Dept. Internal Medicine, Rheumatology Clinic, UHC Mother Teresa, Tirana; ${ }^{3}$ Dept. Cardiovascular Diseases, Cardiovascular Reanimation Clinic, UHC Mother Teresa, Tirana, Albania

\subsection{6/lupus-2020-eurolupus.53}

Background Antiphospholipid Syndrome (APS) is a known cause of thrombotic disorders, including Acute Myocardial Infarction (AMI). Although its incidence in AMI patients is not known, it may be an important factor in precipitating infarction, especially in younger adults.

Methods This is a case-control study consisting in 73 patients with Acute Myocardial Infarction, hospitalized at Cardiovascular Reanimation Clinic from 10 December 2015- 10 October 2019. All patients included in the study were from 23 to 50 years old. In the case-group were included 24 patients with Antiphospholipid Syndrome and Acute Myocardial Infarction, and 49 patients were included in the control group, which consisted only in patients with AMI, with no presence of APS. In every patient were gathered data such as complete blood count + ESR, Antinuclear Antibodies (ANA), Anti-cardiolipin antibodies (ACA), C3 and C4 complement fraction, Anti-ds-DNA, CRP and Ejection Fraction (EF) in echocardiography. Female patients were asked about their abortion history. As recommended in the guidelines, in positive results for APS, ACA levels were repeated after 12 weeks in order to establish the diagnosis. All data were gathered and statistically analyzed using Excel 2010 and IBM SPSS.

Results After comparing all gathered data, it was found that the patients with APS and AMI had a more significant tendency to have C3 hypocomplementemia $(p=0.006)$, thrombocytopenia $(\mathrm{p}=0.002)$, a lower ejection fraction on transthoracic echocardiography $(p=0.04)$ and a more elevated number of abortions before acute myocardial infarction $(p=0.03)$ in comparison to the controls.

Conclusions From our study it was found that APS is not rare in young adults with AMI. It should be always suspected in young patients with no cardiovascular risk factors and there may be a characteristic clinical and laboratory picture in patients with AMI, which may suggest the APS diagnosis.

Acknowledgements The authors declare no conflict of interest.

\section{P5 RECOGNIZING THE DISEASE, TREATING THE PATIENT: A MIXED-METHOD EVALUATION OF CARE FOR THE ANTIPHOSPHOLIPID SYNDROME (APS) IN THE NETHERLANDS}

\footnotetext{
${ }^{1}$ Mirthe J Klein Haneveld, ${ }^{1}$ Caro Lemmen, ${ }^{1}$ Tammo Brunekreef, ${ }^{2}$ Marc Bijl, ${ }^{3}$ Gerard Jansen, ${ }^{4}$ Karina de Leeuw, ${ }^{1}$ Julia Spierings, ${ }^{1}$ Maarten Limper, on behalf of the ARCH study group. ${ }^{1}$ Dept. of Rheumatology and Clinical Immunology, University Medical Centre Utrecht, Utrecht; ${ }^{2}$ Dept. of Internal Medicine and Rheumatology, Martini Hospital, Groningen; ${ }^{3}$ Dept. of Hematology, Erasmus MC, University Medical Center Rotterdam, Rotterdam; ${ }^{4}$ Dept. of Rheumatology and Clinical Immunology, University Medical Center Groningen, Groningen, The Netherlands
}

10.1136/lupus-2020-eurolupus.54
Purpose This study aims to gain insight into the care provided to patients with antiphospholipid syndrome (APS) in The Netherlands and to identify areas for improvement from the perspective of both patients and clinicians.

Methods APS care was evaluated using qualitative and quantitative methods. Perspectives on APS care were identified using semi-structured interviews with medical specialists, patient focus groups and a cross-sectional, online patient survey. In order to examine differences in medical practice, medical records were reviewed retrospectively regarding clinical and laboratory manifestations, pharmacological treatment and management of disease in six hospitals throughout The Netherlands.

Results Fourteen interviewed medical specialists, fourteen focus group participants and 79 survey respondents participated in the study. 237 patients were included in medical record review. Only one-third of patients were diagnosed with APS within three months after entering specialist care. Diagnosis and management varied between centres and specialists. Almost $10 \%$ of triple positive patients did not receive any treatment at the time of medical record review. Major challenges according to specialists and patients were poor recognition of APS by health care professionals, fragmentation of care and lack of accessible, reliable patient education and psychosocial support.

Conclusion This study describes delayed diagnosis, variability in management strategies and a burden placed on patients to orchestrate their own care in APS care in The Netherlands. Of note, almost $10 \%$ of triple positive patients did not receive any treatment, despite this group of patients being at the highest risk of recurrent thrombosis.

Acknowledgements This work was supported by the Arthritis Research and Collaboration Hub (ARCH) Foundation.

\section{P6 RITUXIMAB EFFICACY IN CAPS}

Olena Yakymenko, Viktoria Vasylets, Viktor Klochko, Vladimir Savytskyi, Natalya Tikhonchuk. Rheumatological Dept., Multifield Medical Center of Odessa national medical university, Odesa, Ukraine

\subsection{6/lupus-2020-eurolupus.55}

Background Catastrophic antiphospholipid syndrome (CAPS) thrombotic microvasculopathy, characterized by involvement of systems and organs with formation of their insufficiency. Therapy of glucocorticoids and immunosuppressants with plasmapheresis and intravenous immunoglobulin demonstrates lack of effectivity. Currently, there are reports of rituximab effectiveness in APS and CAPS, but they are not numerous, especially CAPS.

Methods Assessment of the rituximab effectiveness in patients with resistant CAPS. We present the treatment results of two patients with CAPS, who were treated in our clinic.

Results Patient V., 21, was admitted in the early postpartum period. Diagnosis: Systemic lupus erythematosus, positivity for ANA: ds-DNA, Sm. Secondary antiphospholipid syndrome (positivity for $\beta 2$-glycoprotein-1, cardiolipin, lupus anticoagulant), catastrophic APS with multiorgan failure: epilepsy, sopor, psychosis; acute renal injury, signs of acute respiratory distress syndrome and hypocoagulation. SLEDAI score was 56 points, GAPSS - 17 points. Pulse therapy with methylprednisolone, cyclophosphamide, plasmapheresis and intravenous immunoglobulin wasn't effective enough. Rituximab course $375 \mathrm{mg} / \mathrm{m}^{2} 1$ time per week for 4 weeks was administered. Manifestations 
of CAPS in multiorgan failure form regressed 14 days after the first administration, SLEDAI score decreased to 32 points, GAPSS to 4 points. After 4 weeks complete B-cell depletion was achieved. A month after the course of rituximab she achieved remission, which lasts 4 years already. ANA and antiphospholipid antibodies weren't detected.

Patient E., 20, with primary APS, cardiolipin positivity, $\beta 2-$ glycoprotein-1 and lupus anticoagulant, thrombocytopenia, livedo reticularis; CAPS-like thrombotic microangiopathy type with damage to cerebral vessels, lung vessels, recurrent pulmonary embolism for six months, deep leg vein thrombosis. GAPSS activity before treatment was 17 points. Pulse therapy wasn't performed. Therapy rituximab $375 \mathrm{mg} / \mathrm{m}^{2} 1$ time per week, 4 weeks was administered. Multiple organ failure also regressed 10-14 days after 1 administration of rituximab. GAPSS score decreased to 10 points. Incomplete B-cell depletion was achieved.

Conclusion Thus, rituximab demonstrated high effectiveness in CAPS in both cases. Rituximab allowed to reach multiple organ failure regression and a persistent effect was achieved.

\section{P7 RIVAROXABAN MAY TRIGGER CATASTROPHIC ANTIPHOSPHOLIPID SYNDROME}

${ }^{1}$ Romain Stammler, ${ }^{1}$ Paul Legendre, ${ }^{2}$ Patrice Cacoub, ${ }^{1}$ Philippe Blanche, ${ }^{2}$ Jean Charles Piette, ${ }^{1}$ Nathalie Costedoat-Chalumeau. 'Internal Medicine Dept., AP-HP Cochin Hospital, Paris; ${ }^{2}$ Internal Medicine Dept., AP-HP La Pitié-Salpêtrière Hospital, Paris, France

\subsection{6/lupus-2020-eurolupus.56}

Background Catastrophic antiphospholipid syndrome (CAPS) is the most severe complication of antiphospholipid syndrome (APS). Vitamin K antagonists (VKAs) are the reference treatment for preventing relapsing thrombotic complications in patients with APS, but direct oral anticoagulants (DOACs), such as rivaroxaban, are nonetheless sometimes used in patients with antiphospholipid antibody profiles or APS. Recent studies showed that DOACs were associated with more arterial thromboses among patients with APS. The potential role of DOACs as a trigger factor of CAPS is not known.

Methods We describe two patients who developed a CAPS in the week following the institution of rivaroxaban.

Results We report the onset of definite CAPS in the week following introduction of rivaroxaban treatment in two patients, one with APS and the other with antiphospholipid laboratory findings only. Both were triple positive for antiphospholipid antibodies. The affected organs were the heart, kidneys, skin, and liver for Patient 1 , and the heart, kidneys, skin, adrenal gland, and central nervous system for Patient 2. The causative role of rivaroxaban is highly probable given that (1) CAPS occurred rapidly after this treatment was started, (2) an alternative trigger factor was found in Patient 1 only (a colonoscopy), and (3) Patient 1 had been clinically stable for 18 years with VKA as anticoagulant treatment, while Patient 2 did not have APS and had had no symptoms for 4 months (rivaroxaban had been introduced because at a scheduled visit, she reported neurological symptoms that occurred four months before and were retrospectively compatible with a brain transient ischemic accident). One similar case was reported in 2017, also following the introduction of rivaroxaban, in a patient with triple positive venous thrombotic APS who was stable for years on warfarin and who developed definite CAPS (involvement of the myocardium and adrenal glands as well as a pulmonary embolism) in the week after rivaroxaban 20 mg daily replaced warfarin to meet the patient's desire for a less burdensome treatment. ${ }^{1}$ Finally, in the randomized study published by Ordi-Ros et al, one of the patients treated with rivaroxaban developed a CAPS. ${ }^{2}$

Conclusions These two cases, as well as two previous reported cases, underline the importance of avoiding DOACs in patients with APS, especially those triple positive for antiphospholipid antibodies. VKAs must remain the reference anticoagulation treatment in this setting.

\section{REFERENCES}

1. Crowley MP, Cuadrado MJ, Hunt BJ. Catastrophic antiphospholipid syndrome on switching from warfarin to rivaroxaban. Thromb Res 2017; 153; 37-9.

2. Ordi-Ros J, Saez-Comet L, Pérez-Conesa M, Vidal X, Riera-Mestre A, Castro-Salomo $A$, et al. Rivaroxaban versus vitamine $K$ antagonist in antiphospholipid syndrome: a randomized noninferiority trial. doi: 10.7326/M19-0291. [Epub ahead of print]; 2019

\section{P8 REFRACTORY THROMBOCYTOPENIA IN A PATIENT WITH SYSTEMIC LUPUS ERYTHEMATOSUS AND SECONDARY ANTIPHOSPHOLIPID SYNDROME}

${ }^{1,2}$ Rita Torres, ${ }^{1}$ Maria João Gonçalves, ${ }^{1,2}$ Nuno Gonçalves, ${ }^{1,2}$ Santiago Manica, ${ }^{1,2}$ Agna Neto, ${ }^{1,2}$ José Marona, 'Manuela Costa, ${ }^{1,2}$ Fernando M Pimentel-Santos, ${ }^{1,2}$ Jaime C Branco. ${ }^{1}$ Rheumatology Dept., Centro Hospitalar Lisboa Ocidental, Hospital de Egas Moniz, Lisboa; ${ }^{2}$ NOVA Medical School, Universidade NOVA de Lisboa, Lisboa, Portugal

10.1136/lupus-2020-eurolupus.57

Background Thrombocytopenia is a frequent hematological manifestation in patients with systemic lupus erythematosus (SLE), usually treated with glucocorticoids, immunosuppressants (such as azathioprine and cyclophosphamide), intravenous immunoglobulin (IVIG) or splenectomy.

Case report A 44-year-old woman diagnosed with SLE and secondary antiphospholipid antibody syndrome was admitted in our hospital, in July 2018, presenting severe renal and haematological involvement. She underwent treatment with methylprednisolone, followed by prednisolone, and mycophenolate mofetil, with no response. In August 2018, the patient started haemodialysis, human immunoglobulin (transient response) and plasmapheresis due to evidence of secondary thrombotic microangiopathy. The refractory severe thrombocytopaenia and lupus nephritis, justify two cycles of rituximab - platelet count rising lasted for five months. Seven months later, the patient presented pancytopenia and due to high hemorrhagic risk, there was a switch from warfarin to LMWH (prophylactic dosing). One month later, anti-coagulation was suspended due to spontaneous intracranial bleeding. In August 2019, the patient had an ischemic stroke of the right occipital lobe, secondary to APS, under prednisolone and hydroxychloroquine, and with platelet count of $24.000 / \mathrm{uL}$. Because of the bleeding risk, no anti-aggregation was instituted, and she started tacrolimus - the initial good response disappeared two weeks later. After consulting a rheumatology board, tacrolimus was kept and rituximab repeated, regardless of the hypogammaglobulinemia and no lymphocytes CD19+ count.

Conclusion We experienced a severe case of secondary thrombocytopenia, that was refractory to multiple therapeutic agents. A reasonable response was obtained under rituximab. Tacrolimus is kept due to the reported cases of late effect. Ischaemic stroke is a challenging condition in patients thrombocytopenic and further clinical guidance is warranted. 